\title{
Involvement of carotid artery by a chordoma: Surgical pitfall
}

Sir,

A 70-year-old female patient admitted to our hospital with complaints of transient ischemic attacks (TIAs) exacerbated by head-neck movements, pain and a pulsatile neck mass on the right. A huge mass, extending from the C3-C4 vertebral bodies to the right common and internal carotid arteries (RCCA, ICA) was detected on the cervical Magnetic Resonance Imaging (MRI). The arteries were displaced, compressed and kinked by the mass, as shown by Magnetic Resonance Angiography [Figure 1]. Doppler examination revealed low-flow velocity and diameter reduction in these arteries. The mass was diagnosed as chordoma by needle aspiration biopsy. Most of the tumor mass was removed $(4 \times 6.5 \times 3.5 \mathrm{~cm})$ in the first operation. The tumor was separated from the carotid artery applying deep adventitial dissection leaving the vessel intact. All neurological symptoms were abolished after the operation. One month later a second operation was performed with neurosurgeons, through a postero-lateral incision, removing the rest of the mass, reconstructing the vertebral corpus and controlling the vertebral arteries, which were intact. Homologous iliac crest bone 
graft was utilized for vertebral reconstruction. Titanium plate was used for the fixation of cervical spine.

The patient has done well afterwards, but in the second year of her follow-up a recurrent mass was diagnosed at the same location involving the RCA bulb/right ICA. So a third operation was undertaken, but this time the mass and carotid artery within were removed completely together. The carotid artery was reconstructed with No $6 \mathrm{~mm}$ polytetrafluoroethylene (PTFE) graft. Pathologic studies from the specimens found tumor cell scattered at several sites on the wall of the artery [Figure 2].

Chordoma is a rare bone neoplasm $(1-4 \%$ of bone malignancies), which originates from the embryonic notochord. ${ }^{[1]}$ Cervical chordoma, arising in the cervical spine, grows progressively into the soft tissue of the neck. The symptoms become evident by the compression of the chordoma to the adjacent structures. Surgical resection of the tumor is curative if completely removed, due to its low-grade malignancy and non-metastasis characteristics. ${ }^{[2]}$ The two consecutive operations (with anterior and posterior approaches) before the recurrence operation, were devised to fully remove the tumor, for complete resection of the chordoma and reconstructing the cervical spine and carotid artery in our patient.

Carotid arteries may be complicated by the neighboring tissue tumors. These tumors may cause compression, kinking, flow restriction, turbulent jet flow, intimal ulcerations and micro thromboembolism into the carotid artery circulation. ${ }^{[3]}$ Carotid artery involving by chordomas are rare cases, however, a few reports have been presented in the literature ${ }^{[1]}$ Recurrence rate of chordoma may reach up to $30 \%$, predominantly from the local surgical area ${ }^{[4]}$ However, for this case in which the huge tumor spanned the whole neck from posterior to anterior, a two-staged operation was planned. Priority was given to the carotid artery reconstruction where the neurological findings were prominent. In the first

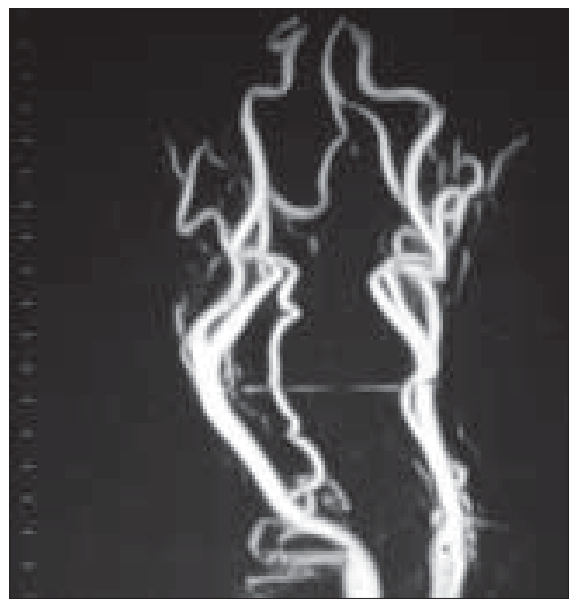

Figure 1: Displacement and kinking of right ICA are shown by MR angiography

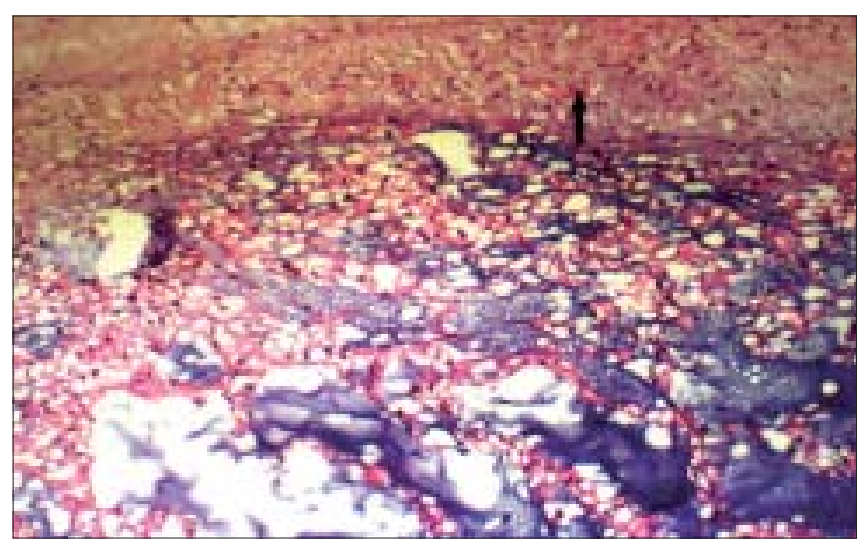

Figure 2: The intricate relation of carotid artery and chordoma can be seen on the microscopic view. (Shown by Arrow) (H\&E, x40)

stage, the chordoma was removed from the artery using deep periadventitial (white-line) dissection, relying on its low-grade; malignancy, potential for metastasis and invasive features. This case revealed that the recurrence is highly possible due to the chordoma cells remaining residual into the artery wall.

In our opinion the carotid artery complicated by chordomas should be replaced with grafts due to the high recurrence and infiltration capability of chordomas.

\section{Mehmet Raşit Güney, Bulend Ketenci, Batuhan Ozay, Nihat Ozer ${ }^{1}$, Serdar Cimen}

Departments of Cardiovascular Surgery and ${ }^{1}$ Cardiology, Siyami Ersek Chest and Cardiovascular Surgery Centre, Istanbul, Turkey. E-mail: bulendketenci@gmail.com

\section{References}

1. Leone A, Cerase A, Tarquini E, Mule A. Chordoma of the low cervical spine presenting with Horner's syndrome. Eur Radiol 2002;12:S43-7.

2. Boriani S, Chevathelley F, Weinstein JN. Biagini R, Campanacci L, De Iure F, \& al. Chordoma of the spine above the sacrum: Treatment and outcome in 21 cases. Spine 1996;21:1569-77.

3. Freeman SB, Hamaker RC, Borrowdale RB, Huntley TC. Management of neck metastasis with carotid artery involvement. Laryngoscope 2004;114:20-4.

4. Fagundes MA, Hug EB, Liebsch N., Daly W, Efird J, Munzenrider JE. Radiation therapy for chordomas of the base of skull and cervical spine: Patterns of failure and outcome after relapse. Int J Radiat Oncol Biol Phys 1995;33:579-84.

Accepted on 25-02-2008 